\title{
THREE-DIMENSIONAL RECONSTRUCTION OF THE TREE CANOPY FOR ASSESSMENT OF LEAFS
}

\author{
Berk, P., StajnKo, D., LAKOTA, M. \& BelsaK, A.
}

Abstract: A research project based on a principle of a laser measuring system was designed to determine geometric structure of the tree canopy (number of leafs and shape of the tree canopy) in the orchard, namely, from the left and right side of the canopy. In the research project advanced laser technology involved a laser measurement sensor LMS111 and a Labview 2013 software package, through which a data acquisition from a laser measuring sensor was enabled. Accurate assessment of proportion number of leafs on the selected tree canopy were obtained on the basis of automated imaging system and connected to laser measurements, which represented the number of the reflected laser beam from the leaf area of the tree canopy. Analysis between the measured proportion the manually counted number of leaf and the number of laser beams reflected from the leaf area of tree canopy was made in Matlab R2013a software package and will be included in further research on the mathematical model for continuous process of plant protection products (hereinafter PPP) application on the selected tree canopy in the orchard.

Key words: measurement, sensor, telegram, orchard, tree canopy
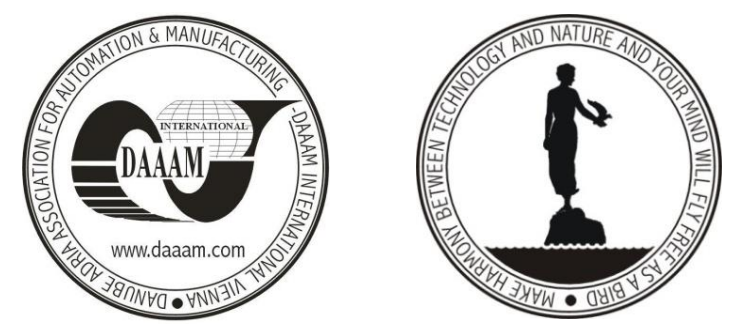

Authors' data: Msc. Berk, P[eter]*; Assoc. Prof. Stajnko, D[enis]*; Assoc. Prof. Lakota, M[iran $]^{*}$; Dr. Belsak, A[les]**, *University of Maribor, Faculty of Agriculture and Life Sciences, Pivola 10, SI-2311, Hoce, Slovenia, **University of Maribor, Faculty of Mechanical Engineering, Smetanova ulica 17, SI-2000, Maribor, Slovenia, peter.berk@um.si, denis.stajnko@um.si, miran.lakota@um.si, ales.belsak@um.si

This Publication has to be referred as: Berk, P[eter]; Stajnko, D[enis]; Lakota, M[iran] \& Belsak, A[les] (2016). Three-Dimensional Reconstruction of the Tree Canopy for Assessment of Leafs, Chapter 06 in DAAAM International Scientific Book 2016, pp.059-068, B. Katalinic (Ed.), Published by DAAAM International, ISBN 9783-902734-09-9, ISSN 1726-9687, Vienna, Austria

DOI: $10.2507 /$ daaam.scibook.2016.06 
Berk, P.; Stajnko, D.; Lakota, M. \& Belsak, A.: Three-Dimensional Reconstruction...

\section{Introduction}

Precise knowledge of geometrical canopy characterization is one of the most important factors for characterization of tree canopy to improve PPP process application in apple orchards. The most important parameter in the PPP process application represents a leaf area of tree canopy or so-called leaf area index (hereinafter LAI). It is defined as the one-sided green leaf area per unit ground surface area in broadleaf canopies. These parameters influence the relationship between the sprayer output and the deposit on the target crop. LAI can be measured manually, but in this case it requires a destructive, time consuming and expensive method including the total defoliation of individual tree and extended laboratory measurements to determine the leaf area on an individual canopy tree. Therefore, a different electronic sensing measurement systems were developed, which made the estimation of leaf area density and tree canopy volume characteristics possible. Byers et al. (1984), and Walklate et al. (2000) found a way to determine geometric structure of tree canopy by means of electronic measurement system, more precisely. However, the canopy varies enormously according to vegetative stage, tree age, varieties, plant density, leaf area, and training type. An electronic measuring systems, which were developed by individual researchers in the past and which we introduce hereinafter are based on an ultrasonic (Stajnko et al., 2012; Berk et al., 2013), stereoscopic and spectral scanning systems (Kelc et al. 2015). In this paper we will limit only to spectral scanning systems.

With spectral measurement systems based on the LIDAR sensors principle, it is possible to measure distances between the sensor and the objects in its vicinity very precisely and quickly. In this way, three dimensional point clouds can be made (elements representing leaves and branches for the selected geometric structure of tree canopies). With appropriate algorithms, point clouds which is represented in Figure 1, can be used to digitally reconstruct and describe the tree structure in an orchard very precisely (Osterman et al., 2013).

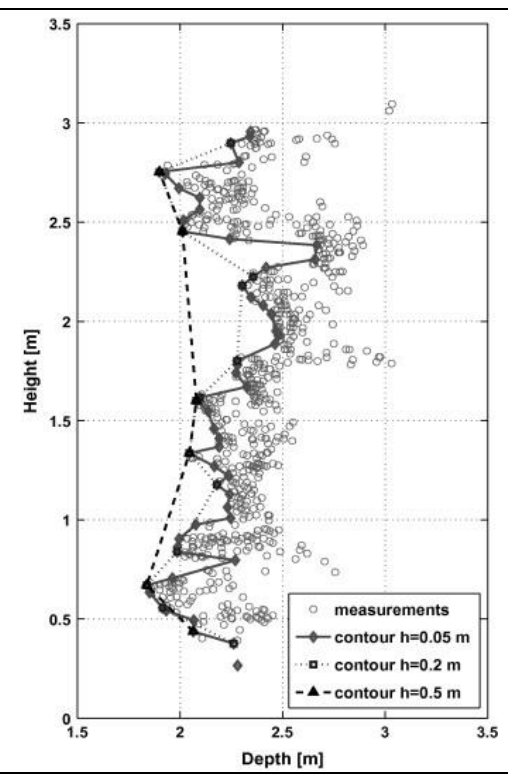

Fig. 1. Digital reconstruction of a tree canopy structure in an apple orchard (Osterman et al., 2013) 
Walklate et al. (2002) used a LIDAR sensor, mounted on a tractor for rapidly characterize crop structure, so that the times of flight of the pulses to the points of interception with the crop, were measured and the range and angle of interception was calculated, shown in Figure 2. Each rotational measurement point sequence was made of 200 range measurements with a scan angle of 100 degrees. Walklate et al. (2002) defined tree-row cross-section by a two-dimension map. Using a laser measurement system (Walklate et al., 2002) are measured and calculated canopy parameters such as: h-maximum height, b-width (i.e. the cross-section by divided by the maximum height) and a-average of the canopy-density (the area of leaf and fruit surface per unit volume of canopy).

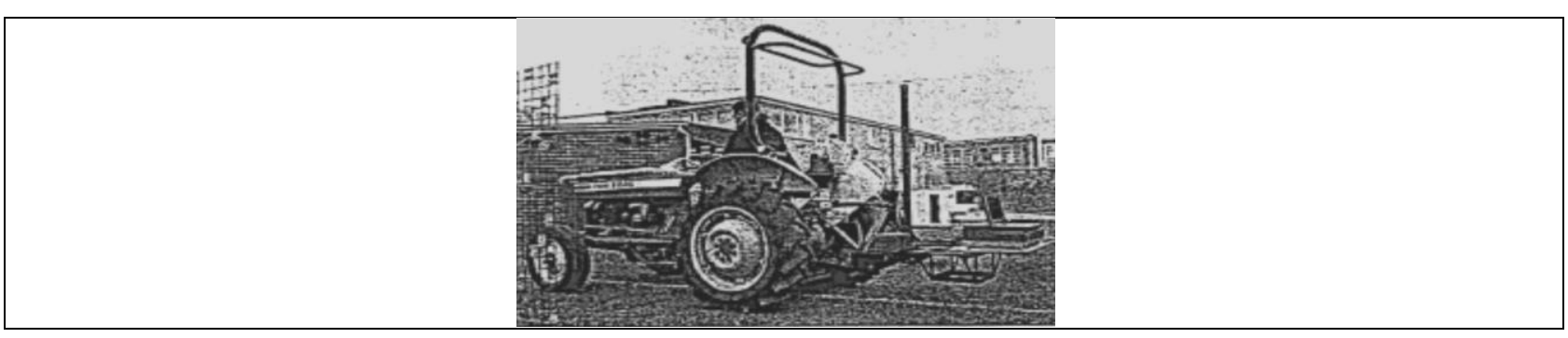

Fig. 2. Side view of the LIDAR system mounted on the rear of a tractor (Walklate et al., 2002)

In perform of canopy characterization measurement (Escola et al., 2013) also used a LIDAR sensor. With help of detection measurement system they determined the cross-sectional area of the tree canopy. The LIDAR sensor was attached to a vertical pole in the front part of the sprayer prototype facing to the rows of trees to be treated. Escola et al. (2013) found that a conventional orchard sprayer treats the two halves of both rows facing the passages; for this reason, the canopy characterization measurement system only measures the half facing the operational side of the sprayer. Measurement system estimated the semi-cross-sectional area of the canopy, every 10$20 \mathrm{~cm}$ along the row from the readings of the LIDAR sensors, Figure 3 . The output of the canopy characterization measurement system, which was represented by canopy semi-cross-sectional area, determined the dosage to be sprayed according to tree canopy variability (Escola et al., 2013).

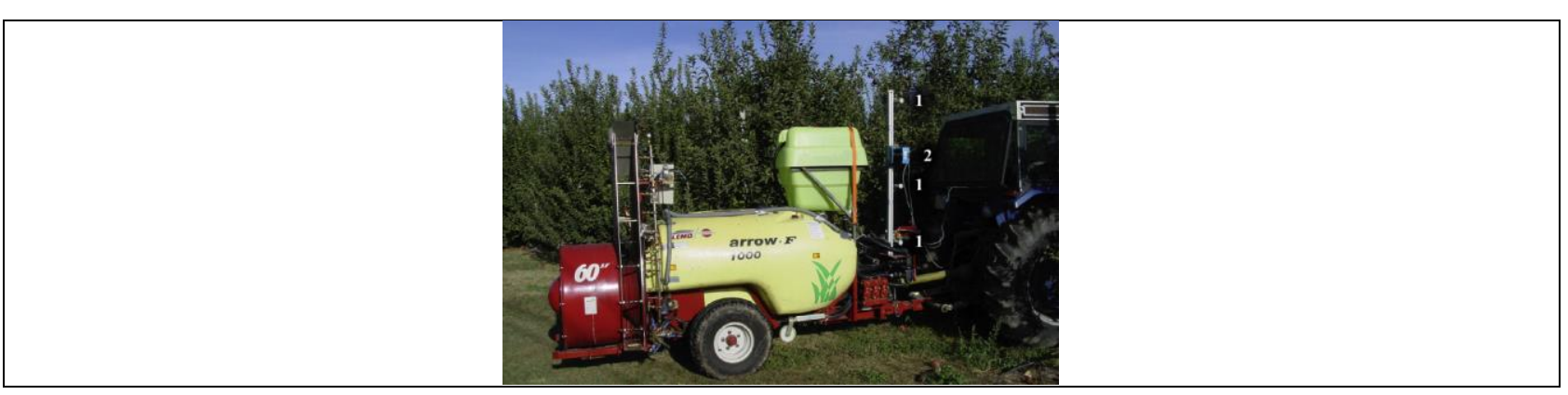

Fig. 3. Variable-rate orchard sprayer prototype implemented with LIDAR sensor (Escola et al., 2013) 
Berk, P.; Stajnko, D.; Lakota, M. \& Belsak, A.: Three-Dimensional Reconstruction...

The usefulness of image registration techniques in mapping and localising a robot in an agricultural environment (orchard) by using readings from a laser range scanner was precisely investigated by Lepej \& Rakun (2016).

\section{Design of electronic sensing system using software and hardware components}

The non-linear geometric structure of the tree canopy in an orchard is influenced by various parameters, among which the following ones may be considered: variety, age, size, pruning, and phenological stages of the trees. A non-linear geometrical structure of the tree canopy can be evaluated with the use of advanced spectral measurement systems, operating on the laser LMS111 sensor principle. With the help of advanced LMS111 a leaf area surface and three-dimensional geometric structure of the individual tree canopy was defined in the orchard. For defining quality process control application of measurement system a TCP/IP connection for the data acquisition from the LMS111 was used, Figure 4. The TCP/IP gives us a possibility to measure and use data from distant locations. The LMS111 sends messages over the interfaces to communicate with a connected host. The entire structure of the data acquisition process was designed with the LabVIEW 2013 software package and hardware components. Measured data from LMS111 were exported to the Excel file and used for later processing. Exported measured data was imported into Matlab R2013a software package so a number of hits a laser beam from leaf area surface and three-dimensional geometric structure of the individual tree canopy can be defined.

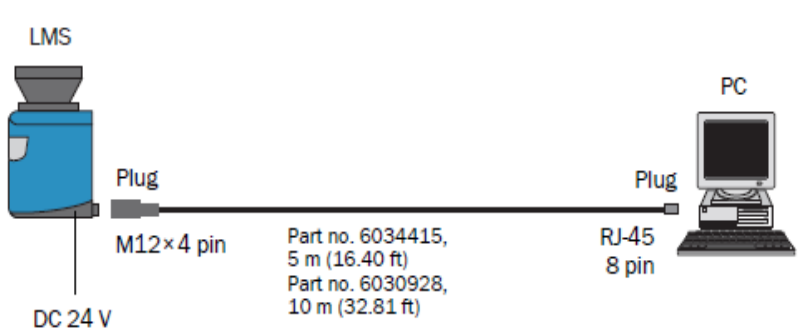

Fig. 4. TCP/IP connection for the data acquisition from the LMS111 sensor

\subsection{Hardware}

The hardware of the electronic measurement system for non-linear geometrical structure of the tree canopy consists of:

- A hardware electronic sensing system was built around the HP Compaq 6830s. A HP 6830s Laptop has a 3 GB DDR2 $800 \mathrm{MHz}$ memory, expandable to 8 GB. Hard Drive has a capacity of $320 \mathrm{~GB}$, supports SMART SATA bus communicates and operates plants in 5400 in a minute.

- LMS 111 laser measurement sensor. The LMS 111 is an electro-optical laser measurement sensor that electro-sensitively scans the perimeter of its surroundings in a plane with the aid of laser beams. The LMS measures its surroundings in two-dimensional polar coordinates. If a laser beam is incident on an object, the position is determined in the form of distance and direction, Figure 5. Scanning 
takes place in a sector of $270^{\circ}$. The scanning range of the LMS111 is maximum 20 $\mathrm{m}$ on light, natural surfaces with an object remission $>13 \%$ (e.g. a white house wall). The emitted laser beams are deflected using a mirror and scan the surroundings in a circular manner. The measurements are triggered at regular steps using an angular encoder. The LMS111 scans with a scanning frequency of 25 or $50 \mathrm{~Hz}$. During this process, a laser pulse and therefore a measurement is triggered after an angular step of $0.25^{\circ}$ or $0.50^{\circ}$.

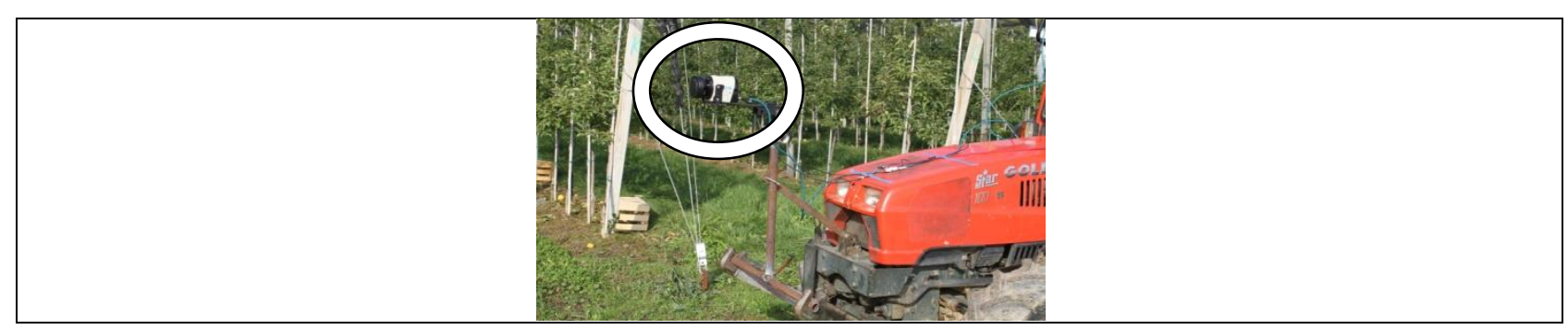

Fig. 5. Measuring principle of the LMS111 in a tree canopy orchard

\subsection{Software package LabVIEW 2013}

LabVIEW is a program development application like various commercial $\mathrm{C}$ or BASIC development systems, or National Instruments LabWindows. However, LabVIEW is different from those applications in one important respect. Other programming systems use text based languages to create lines of code, while LabVIEW uses a graphical programming language, to create programs in block diagram form. LabVIEW uses terminology, icons, and ideas familiar to scientists and engineers and relies on graphical symbols rather than textual language to describe programming actions. For Windows, Macintosh, and Sun, LabVIEW contains application specific libraries for data acquisition and VXI instrument control. LabVIEW also contains application-specific libraries for GPIB and serial instrument control, data analysis, data presentation, and data storage. We can use LabVIEW to communicate with hardware such as data acquisition, vision, and motion control devices, as well as GPIB, PXI, VXI, RS232, and RS485 instruments.

\subsection{Software package Matlab R2013a}

Matlab is a software package designed for numerical calculations, such as arithmetic operations of vectors, differential equations and presentation of results. It was originally designed for solving linear algebra type problems using matrices. Such extensive use of Matlab has since been expanded and now has built-in functions for solving problems requiring data analysis, signal processing, optimization, and several other types of scientific computations. It also contains functions for two-dimensional and three-dimensional graphics and animation. Due to the ease of use Matlab to become a tool, they have become widely used. It is suitable for teaching, research and solves practical problems. MATLAB language has rich data structures and its object oriented. Because Matlab interprets own files, the loss of much valuable time, but it is possible to code $\mathrm{m}$-files to translate and thus significantly speed up the implementation of the program. On the other hand, bottlenecks program, who spend the most time, encoded in any other programming language, such as C. Matlab code can be translated 
Berk, P.; Stajnko, D.; Lakota, M. \& Belsak, A.: Three-Dimensional Reconstruction... into mex-file, it know how to use Matlab as its own m-file, only that translated performed much faster. Matlab has some distinctive advantages:

- quickly and easily writing programs,

- available to receive high-quality tools for visualization,

- program running on multiple operating systems and

- offers extensive facilities for displaying vectors and matrices as graphs, as well as annotating and printing these graphs.

\section{Modeling application of a non-linear geometrical structure of the tree canopy}

A non-linear geometrical structure of the tree canopy was made by using a LabVIEW 2013 software package, which enables to capture data from LMS111 sensor and software package Matlab R2013a, so a three-dimensional virtual model of the tree canopy was modified. A three-dimensional modeling application can be considered as a system with non-linear dynamic characteristic. For this reason, the application included an electronic measurement system, which creates the tree canopy geometrical structure depending on the LMS111 measurements in two-dimensional polar coordinates. Optimization of measurement system for defining geometrical structure of the tree canopy was started with the data acquisition process from the sensor LMS111. Therefore a TCP/IP communication protocol was used, which specify the data to be sent or received. Via communication scheme, representing by Figure 6, messages in hexadecimal format were sent to the sensor LMS111. Responses of the LMS111 with the parameter values information about the distance and reflectivity energy value of the object were saved in an Excel file via communication scheme shown in Figure 6.

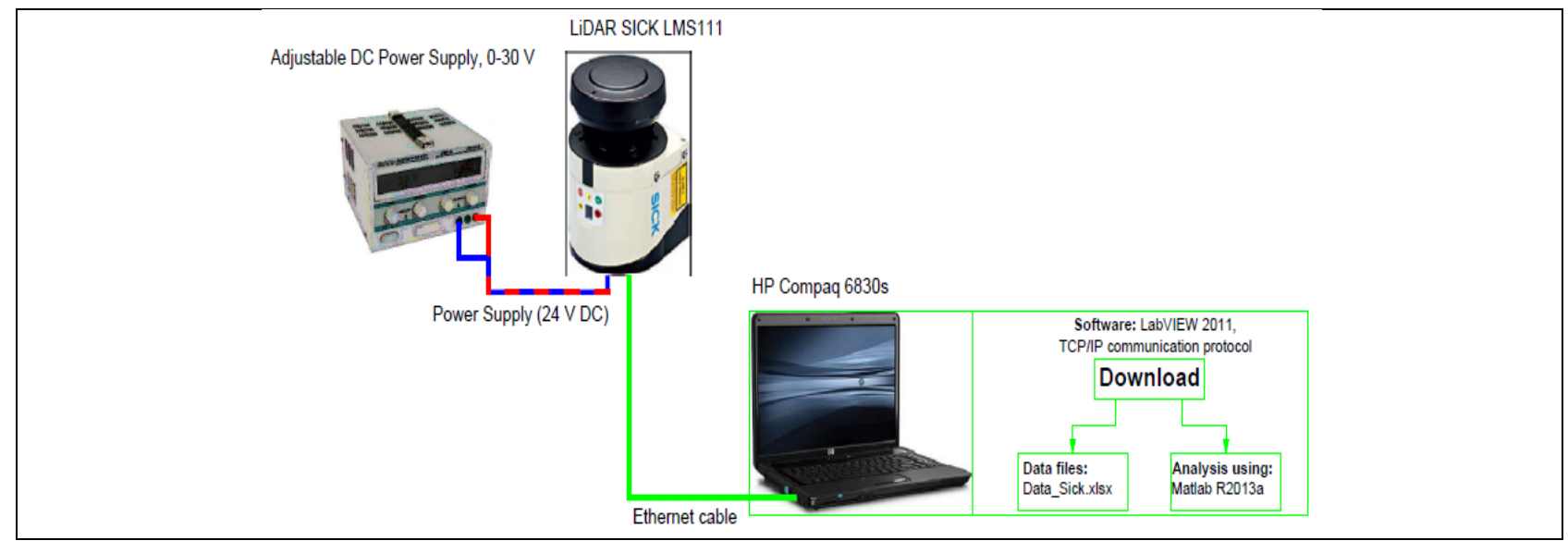

Fig. 6. Communication scheme between LMS111 sensor and host HP Compaq 6830s

A three-dimensional virtual model of the tree canopy was modified by the flow chart algorithm model in Matlab R2013a software package shown in Figure 7. The distance measurement data were imported from Excel file to the Matlab's space, so the distance parameter values exceeded $2.0 \mathrm{~m}$ were excluded from further processing. The next step was to define X, Y, Z-coordinate for each data measurements and to put them into the Cartesian coordinate system. The $\mathrm{X}$-coordinate of each measurement was represented by the equation:

$$
X_{n}=n \cdot v \cdot \Delta t_{n}
$$


where:

$n$ - Nth measurement

$v$ - Constant tractor speed in $\mathrm{kmh}^{-1}$

$\Delta t_{n^{-}}$Time period given by equation:

$$
\Delta t_{n}=t_{n+1}-t_{n}
$$

where:

$t_{n+1^{-}}$Time interval $\mathrm{n}+1$ measurement in mili seconds

$t_{n}$ - Time interval $\mathrm{n}$ measurement in mili seconds

The Y-coordinate of measurement point was represented by the equation:

$$
Y_{n}=\cos \theta_{n} \cdot d_{n}
$$

where:

$\theta_{n^{-}}$Angular step of $0.50^{\circ}$

$d_{n}$ - Distance value in $\mathrm{m}$

The Z-coordinate of each measurement point was represented by the equation:

$$
Z_{n}=n \cdot h_{n}
$$

where:

$n$ - Nth measurement point

$h_{c^{-}}$Height step in $\mathrm{m}$

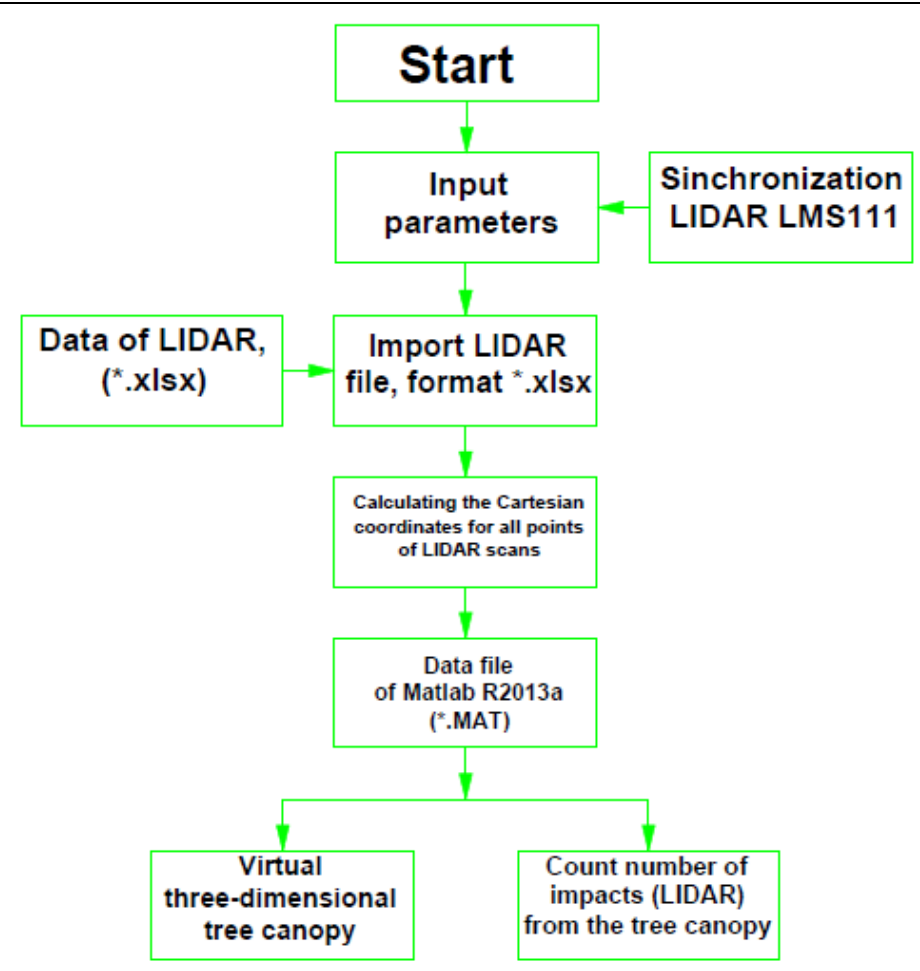

Fig. 7. Flow-chart algorithm diagram for virtual optimization of the tree canopy 
Berk, P.; Stajnko, D.; Lakota, M. \& Belsak, A.: Three-Dimensional Reconstruction...

\section{Results}

Figure 8 shows the two tree canopy in the grove orchard from Faculty of agriculture and life sciences (University of Maribor, Slovenia), for which was made a virtual three-dimensional model, by using software and hardware component. By using a laser measuring system, which is operated by help of a laser LIDAR sensor type LMS 111 was recorded 2 tree canopies from the orchard in the last phenological stage. After completion of the electronic laser measurements were then for each tree canopy manually picked leaf from the left and right half of the tree canopy, and manually calculated the actual number of leaf on each tree canopy. Virtual three-dimensional model of the two tree canopy show Figure 9. Between automated and manually measurements was made an analysis between the number of the reflected laser beams and the actual number of leafs on the left and right sides of the individual tree canopy. We found that automated measurements for the first tree canopy in orchard produced 1883 number of impacts on the right side of the tree canopy and 1605 number of impacts on the left side of the tree canopy from laser measuring system, were was manually counted 417 number of leafs on the right side of the tree canopy and 403 of leafs on the left side of the tree canopy, results are shown in Table 1. Automated measurements for the second tree canopy in orchard produced 2728 number of impacts on the right side of the tree canopy and 1848 number of impacts on the left side of the tree canopy from laser measuring system, where was manually counted 544 number of leafs on the right side of the tree canopy and 522 of leafs on the left side of the tree canopy, results are shown Table 1.

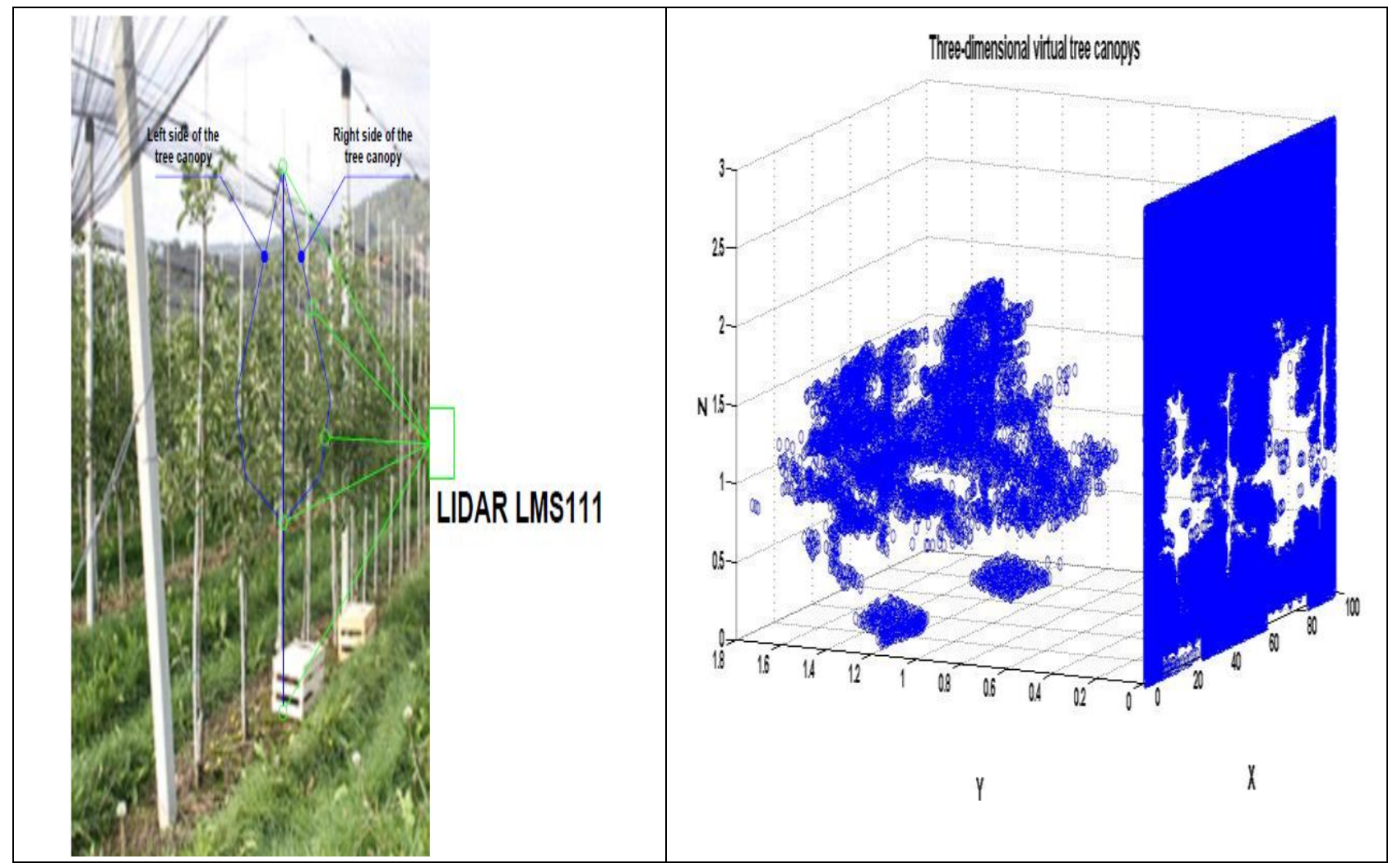

Fig. 8. Individual tree canopy orchard

Fig. 9. Three-dimensional virtual tree in the canopy in the orchard 


\begin{tabular}{|c|c|c|c|}
\hline \multicolumn{4}{|c|}{ Automated and manually measurements } \\
\hline \multicolumn{2}{|c|}{$\begin{array}{l}\text { Automated measurements for the first } \\
\text { tree canopy in orchard }\end{array}$} & \multicolumn{2}{|c|}{$\begin{array}{l}\text { Automated measurements for the second } \\
\text { tree canopy in orchard }\end{array}$} \\
\hline $\begin{array}{l}\text { Number of impacts } \\
\text { on the right side of } \\
\text { the tree canopy }\end{array}$ & $\begin{array}{l}\text { Number of impacts } \\
\text { on the left side of } \\
\text { the tree canopy }\end{array}$ & $\begin{array}{l}\text { Number of impacts } \\
\text { on the right side of } \\
\text { the tree canopy }\end{array}$ & $\begin{array}{l}\text { Number of impacts } \\
\text { on the left side of } \\
\text { the tree canopy }\end{array}$ \\
\hline 1883 & 1605 & 2728 & 1848 \\
\hline \multicolumn{2}{|c|}{$\begin{array}{l}\text { Manually measurements for the first tree } \\
\text { canopy in orchard }\end{array}$} & \multicolumn{2}{|c|}{$\begin{array}{l}\text { Manually measurements for the second } \\
\text { tree canopy in orchard }\end{array}$} \\
\hline $\begin{array}{l}\text { Number of leafs on } \\
\text { the right side of the } \\
\text { tree canopy }\end{array}$ & $\begin{array}{l}\text { Number of leafs on } \\
\text { the left side of the } \\
\text { tree canopy }\end{array}$ & $\begin{array}{l}\text { Number of leafs on } \\
\text { the right side of the } \\
\text { tree canopy }\end{array}$ & $\begin{array}{l}\text { Number of leafs on } \\
\text { the left side of the } \\
\text { tree canopy }\end{array}$ \\
\hline 417 & 403 & 544 & 522 \\
\hline
\end{tabular}

Tab. 1. Results for automated and manually measurements.

On the basis of automated and manually measurements, we can make in further research correlation between number of leaf per tree canopy and the number of hits represented by the reflected laser beams from the tree canopy, however we need recorded for this analysis more than two tree canopy in the orchard. More precise evaluation of the tree canopy will in the future to do with precision Dex-i multicriteria method (Vindis et al. (2012)).

\section{Conclusions}

In the research group we have so far have managed to develop a laser measuring system through which can be estimated characterization (the number of leafs and virtual shape of the tree canopy) of the tree canopy in the apple orchard. The research results confirm that by using an electronic laser measurement system can defining a non-linear geometrical structure of the tree canopy, where usually other measurement sensing systems fails to produce satisfactory results. The laser measurement system was used for transferring data from the appropriate system environment to the virtual system environment. Since this is a very complex system, a special software package LabVIEW and Matlab for modeling geometrical applications were used. Laser measurement system proved to be a very good choice for modeling three-dimensional geometrical structures, so the measurements were very precise and appropriate for virtual three-dimensional modeling of tree canopy.

So far the measurement system for modeling the three-dimensional geometrical structure and defining the number of leafs on the tree canopy was made. Further research will be focused on estimate the size of the leaf area surface on the tree canopy and development automated control system for the PPP process control in the apple orchard. Automated control system will based on laser measurements and software algorithm programmed via control logic system and with which can be able to continuously control of the PPP process in the orchard. Using this application technique may in the future improve the deposit of PPP's (coefficient of variation (\%)) on the leaf surface of the tree canopy with minimal reliance drops losses of PPP's on the soil and in the surrounding orchard area. 
Berk, P.; Stajnko, D.; Lakota, M. \& Belsak, A.: Three-Dimensional Reconstruction...

\section{References}

Byers, R.E.; Lyons Jr., C.G.; Yoder, K.S.; Horsburgh, R.L.; Barden, J.A. \& Donohue, S.J. (1984). Effect of apple tree size and canopy density on spray chemical deposit, Hortscience, vol. 19, pp. 93-94

Walklate, P.J.; Richardson, G.M.; Cross, J.V. \& Murray, R.A. (2000). Relationship between orchard tree crop structure and performance characteristics of an axial fan sprayer, Aspects of Applied Biology, vol. 57, pp. 285-292

Osterman, A.; Godesa, T.; Hocevar, M.; Širok, B. \& Stopar, M. (2013). Real-time positioning algorithm for variable-geometry air-assisted orchard sprayer, Computers and electronics in agriculture, vol. 98, pp. 175-182

Walklate P. J.; Cross J. V.; Richardson, G. M.; Murray, R. A. \& Baker, D. E. (2002). Comparison of different spray volume deposition models using LIDAR measurements of apple orchards, Biosystems Engineering, vol. 82 (3), pp. 253-267

Escola, A.; Rosell-Polo, J.R.; Planas, S.; Gil, E.; Pomar, J.; Camp, F.; Llorens, J. \& Solanelles, F. (2013). Variable rate sprayer. Part 1 - Orchard prototype: Design, implementation and validation, Computers and Electronics in Agriculture, 95, pp. 122135

Lepej, P. \& Rakun, J. (2016). Simultaneous localisation and mapping in a complex field environment, Biosystems engineering, vol. 150, pp. 160-169

Berk, P.; Rakun, J.; Lakota, M. \& Muškinja, N. (2013). The influence of distance ultrasonic density meassurements, DAAAM International scientific book 2013, pp. 637-646

Vindiš, P.; Stajnko, D.; Berk, P. \& Lakota, M.(2012). Evaluation of energy crops for biogas production with a combination of simulation modeling and Dex-i multicriteria method, Polish journal of environmental studies, vol. 21 (3), pp. 237-244

Kelc, D.; Vindiš, P. \& Lakota, M. (2015). Measurements of photosynthesis and transpiration of apple trees, DAAAM International scientific book 2015, pp. 199-208 Stajnko, D.; Berk, P.; Lešnik, M.; Jejčič, V.; Lakota, M.; Štrancar, A., Hočevar, M. \& Rakun, J.(2012). Programmable ultrasonic sensing system for targeted spraying in orchards, Sensors, vol. 12 (11), pp. 15500-15519

https://mysick.com/saqqara/get.aspx?id=im0031331 (Accessed on: 25.08.2016) http://www.ni.com/pdf/manuals/373427j.pdf (Accessed on: 25.08.2016) http://www.mathworks.com/help/pdf_doc/matlab/getstart.pdf (Accessed on: 25.08.2016) 